\title{
Experimental investigation on the effect of platform heating on the dynamic mechanical properties of selective laser manufacturing of AlSi10Mg alloy
}

\author{
Ben Amir ${ }^{1}$, Eyal Grinberg ${ }^{2}$, Yuval Gale ${ }^{3}$, Oren Sadot ${ }^{1}$, and Shmuel Samuha ${ }^{2}$ \\ ${ }^{1}$ Department of Mechanical Engineering, Ben-Gurion University of the Negev, Beer-Sheva 84105, \\ Israel \\ ${ }^{2}$ Department of Materials Engineering, NRCN, P.O. Box 9001, Beer-Sheva 84190, Israel \\ ${ }^{3}$ Additive Manufacturing Center of Excellence, Technologies Division, Israeli Aerospace Industries
}

\begin{abstract}
Additive manufacturing (AM) is one of the emerging promising technology technologies of manufacturing prototypes. The process of AM is based on melting powder by an energetic beam layer by layer to create a three-dimensional body. One of the lightweight alloys that is being used for $\mathrm{AM}$ is AlSi10Mg. The process of manufacturing is controlled by several tens of parameters most of which are determined by the machine manufacturer. One of the important parameters is the building platform temperature. In the present study we took samples from different heights of the building platform and measured the dynamic mechanical properties of each sample. It was noted that after a stress relief treatment (SRT) the difference in the static and dynamic mechanical properties along the building direction changed differently. The dynamic mechanical properties of samples that were fabricated in proximity to the building platform did not change after the SRT, while the mechanical properties of the samples that were fabricated far from the platform changed dramatically and became like those that were fabricated near the building plate.
\end{abstract}

\section{Introduction}

The additive manufacturing (AM) technology is one of the promising processes that will dramatically affect our way of life. The technology most applied is based on melting a powder by intense laser beam and creating a solid structure from the powder. In the laser powder bed fusion (LPBF) process the model is constructed layer by layer; each successive layer is made by applying a thin powder layer of a metallic powder and selectively melting it to create a solid new layer after solidification. Various metallic powders are used for AM such as titanium, steel, nickel, and aluminium alloys. The low weight and high strength of the aluminium alloys make them very applicable for the aerospace and automotive sectors. One of the most common $\mathrm{Al}$ alloys used for $\mathrm{AM}$ is AlSi10Mg near the eutectic point. The microstructure of the product made by AM technology is affected by the superheating and undercooling of the melting beads. The effort to relate the mechanical properties to the manufacturing parameters is extremely important and has been investigated by many $[1,2$, $3,4]$. In all those studies only the static mechanical properties were measured. It was found the the most influential parameters are: laser power, scanning velocity, scanning pattern and hatching distance. Because the laser-based fabrication proccess involves rapid superheating and undercooling, considerable inner stresses are present in the product. Those affect the mechanical properties as well. To reduce the inner stresses heat treatment is often used. The unique microstructure of the AM product is inhomogeneous, therefore it is expected that the 
mechanical behaviour of the product will be dependent on the load direction. It is of great importance to better understand the dynamic mechanical behaviour of the AD product. Also, there are numerous studies on the static mechanical behaviour; however, there are very limited studies on the effect of the manufacturing on the dynamic mechanical properties [5]. In a previous study [6] we measured the strength- strain curve of the AlSi10Mg alloy by Split Hopkinson Pressure Bar (SHPB) at different strain rates while loading the sample along and perpendicular to the building direction. We found that there are significant differences of about $10 \%$ in the yield strength. Moreover, we noted that the strain rate plays an important role. In a later study by our group [7] an effort was made to find the effect of the manufacturing process on the dynamic behaviour. In that study, samples from two different $3 \mathrm{D}$ printers were tested. The manufacturing parameters of the two printers are different; therefore the micro-structure of the produce is different. We succeeded in relating the dynamic behaviour to the micro-structure.

Based on our previous studies regarding the residual stresses in the AM product we asked ourselves: what is the effect of the building platform on the dynamic mechanical properties? During the manufacturing processes the powder has been placed layer by layer on the building platform. The platform is heated to about $200^{\circ} \mathrm{C}$, which reduces the product porosity and improves the manufacturing process. Moreover, a constant heat applied to the product can act as heat treatment and therefore change the mechanical static and dynamic behaviour. In the present study we investigate the effect of the hot building platform on the dynamic mechanical properties and try to relate this behaviour to the microstructure of the product. To do so, we manufactured samples for SHPB taking from different distances from the building platform with several orientations. The samples from the different heights were dynamically loaded along and perpendicular to the building orientation. Static and dynamic measurements were performed together with characterization techniques such as XRD, microstructural characterization and micro-texture analysis.

The paper is organized as follows: first, the fabrication of the samples will be described, followed by a short description of the SHPB system. The mechanical behaviour followed by the characterization results will be presented next, followed by some concluding remarks.

\section{Methods}

\subsection{Sample fabrication}

The Israeli Aerospace Industries (IAI) fabricated the samples for the research. A raw AlSil0Mg powder with $20-63 \mu \mathrm{m}$ fracture size was used. The powder was melted selectively by two $\mathrm{Yb}$ lasers $(\lambda=1070 \mathrm{~nm})$ at a power of $400 \mathrm{~W}$. The focal diameter of the beam was set between $80-115 \mu \mathrm{m}$ while the building platform temperature was set at $200^{\circ} \mathrm{C}$.

Scanning speed of the laser was $1650 \mathrm{~m} / \mathrm{s}$ with hatching distance of $130 \mu \mathrm{m}$. the hatching strategy was bi-directional with $67^{\circ}$ rotation between each layer. The samples were divided into two sets, one underwent standard stress relief heat treatment (SR) while the other set did not and remained in as-built conditions (AB). The samples were fabricated in a vertical orientation. In Fig. 1 the fabrication orientation is presented. We manufactured cubic columns with height of $100 \mathrm{~mm}$ and from each we extruded the samples. From each height we machined samples for static and dynamic tests. The heights were marked A-E as illustrated in the figure. 


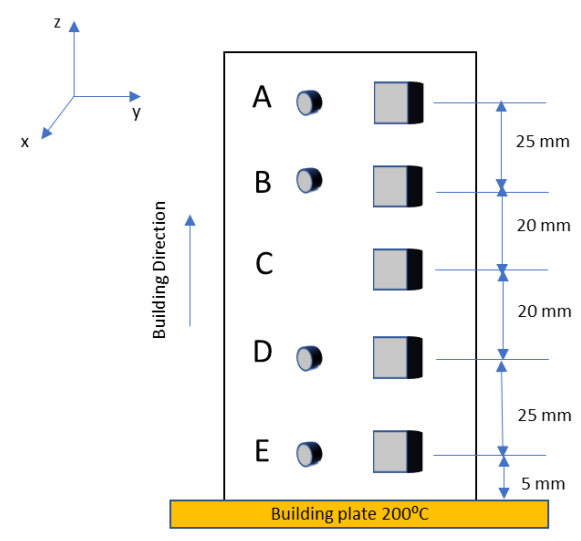

Fig. 1 Schematic of sample extraction from the SLM product.

The samples extracted from the different heights were analysed according to table 1:

Table 1. Analyses performed on the different zones.

\begin{tabular}{|c|c|c|c|c|c|}
\hline Analysis & $\mathbf{A}$ & B & $\mathbf{C}$ & D & $\mathbf{E}$ \\
\hline Density & $\checkmark$ & & $\checkmark$ & & $\checkmark$ \\
\hline Microhardness & $\checkmark$ & & $\checkmark$ & & $\checkmark$ \\
\hline Residual stresses & $\checkmark$ & & & & $\checkmark$ \\
\hline $\begin{array}{l}\text { Texture and } \\
\text { Microstructure }\end{array}$ & $\checkmark$ & & & & $\checkmark$ \\
\hline XRD & $\checkmark$ & $\begin{array}{l}\checkmark \\
*\end{array}$ & & $\begin{array}{l}\checkmark \\
*\end{array}$ & $\checkmark$ \\
\hline SHPB & $\checkmark$ & $\begin{array}{l}\checkmark \\
*\end{array}$ & & $\begin{array}{l}\checkmark \\
*\end{array}$ & $\checkmark$ \\
\hline Fractography & $\checkmark$ & & & & $\checkmark$ \\
\hline $\begin{array}{l}\text { Split Hopkinson } \\
\text { Pressure Bar }\end{array}$ & $\checkmark$ & $\checkmark$ & & $\checkmark$ & $\checkmark$ \\
\hline
\end{tabular}

\subsection{Microhardness}

For near surface hardness measurements, we used a Shimadzu model HMV-2T microhardness machine and Vickers microhardness measurements. We used the following settings: applied load $100 \mathrm{~g}$ with $15 \mathrm{~s}$ indentation dwell time. At least five tests were repeated for each point to increases statistics and reduce noise.

\subsection{Split Hopkinson Pressure Bar tests}

The dynamic response of the SLM-AlSi10Mg samples was tested using the compression split Hopkinson pressure bar at the Laboratory of Dynamic Properties at Ben - Gurion University. The size of the samples was $3.5 \mathrm{~mm}$ height and $7 \mathrm{~mm}$ diameter. The striker velocity can reach up to $21 \mathrm{~m} / \mathrm{s}$ with a bar made from MARG 250 steel. More details about the system can be found in $[6,7]$. 


\subsection{Characterization techniques}

The characterization routine included density measurements, residual stress analysis, microscopy observations, and crystallographic texture analysis. The density was measured using the Archimedes method.

\subsubsection{XRD analysis}

Characterization of the XRD was done on a cross section (side view) of each sample. The sample preparation included electrical discharge cut to expose the building direction and the scanning direction planes, mechanical polish followed by chemical etching.

The macro structure and residual stress were then analysed by an XRD apparatus with Bruker D8 Advance equipped with an open Eulerian cradle, a $\mathrm{Cu} \mathrm{K} \alpha$ source tube (at a voltage of $40 \mathrm{kV}$, and a current of $40 \mu \mathrm{A}$ ), and a PSD detector. For more details about the system and procedure see [8].

\subsubsection{Microstructural characterization and micro-texture analysis}

Scanning electron microscope (SEM) with field emission gun was used to characterize the microstructure. The electron backscattered diffraction (EBSD) technique was employed for microstructural characterization and micro-texture analysis.

\section{Results}

As in the first stage in our study we focused on the mechanical condition of the samples. The density and micro-hardness were measured and are presented in Table 2. The density presented in the table is relative to the density of AlSi10Mg alloy found in the literature.

From the density measurements one should note that the average is close to the tabulated density of AlSi10Mg. However, there is a clear significant trend in the density. Samples that are closer to the building platform (zone E) have higher density with respect to those which are at a greater distance (zone A). This trend is observed both in the AB and SR samples. In contrast to the density measurements, the micro-hardness measurements reveal a different picture. Samples that were treated with the stress relief treatment were found to be unaffected by the distance from the building platform, while there is a clear distance effect for the AB samples. This can be simply explained by the fact that close to the building platform the heat from the platform acts as a stress relief treatment. The same observation was found using XRD measurements.

Table 2. Density, micro-hardness, and residual stress on the different zones

\begin{tabular}{|l|c|c|c}
\hline \multicolumn{1}{c|}{ Analysis } & Zone A & Zone C & Zone E \\
\hline Density AB samples [\%] & $98.2 \pm 0.3$ & $98.7 \pm 0.3$ & $99.4 \pm 0.3$ \\
\hline Density SR samples [\%] & $97.9 \pm 0.6$ & $98.5 \pm 0.5$ & $99.3 \pm 0.3$ \\
\hline Micro-hardness AB samples [HV 0.1] & $121 \pm 4$ & $114 \pm 3$ & $110 \pm 5$ \\
\hline Micro-hardness SR samples [HV 0.1] & $97 \pm 5$ & $96 \pm 5$ & $97 \pm 7$ \\
\hline XDR residual stress [MPa] AB - BD & 76 & & -32 \\
\hline XDR residual stress [MPa] SR - BD & -14.1 & & -13.8 \\
\hline
\end{tabular}


Figure 2 depicts the true-strain true-stress curves measured using the SHPB of four samples: the AB -A and SR-A are the samples fabricated from material far from the building platform (see figure 1) while the AB-E and SR-E were fabricated from material close to the building platform. Each curve is an average of 3 experiments. From the figure, there is a significant difference between the AB-A and AB-E samples. More careful observation reveals that after reaching the yield point $(\varepsilon=0.1)$ there is moderate reduction in the stress. This can be related to accumulating damage in the sample along its compression. One should note that after $\varepsilon=0.8$ there is a drastic decrease in the stress for all the samples; however, there is little difference between the SR samples but significant difference in the AB samples. To further investigate the causes for this behavior we utilized the microscale measurement systems available to us.

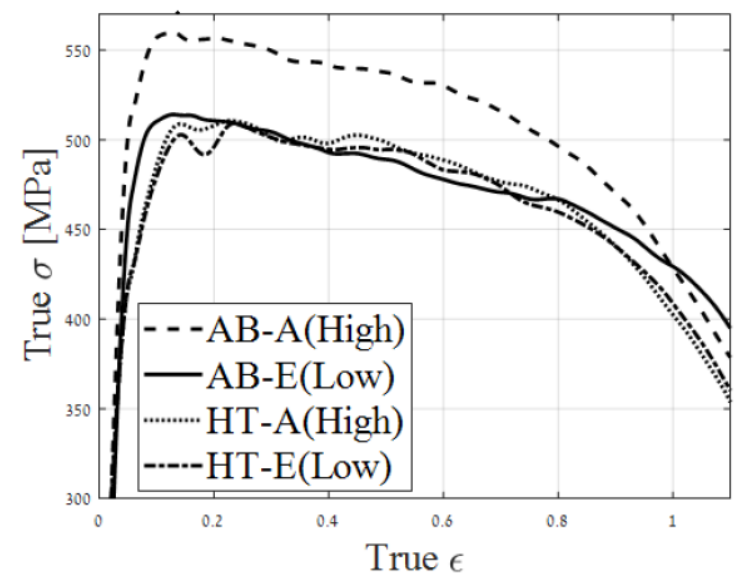

Fig. 2 True-strain true-stress curve obtained from the SHPB system.

The micro and meso scale measurements reveal that there are two completely different micro-cracks present in the high samples (A) vs the low samples (E) close to the building plate. A careful look at the samples reveals an increase in the porosity along the sample height
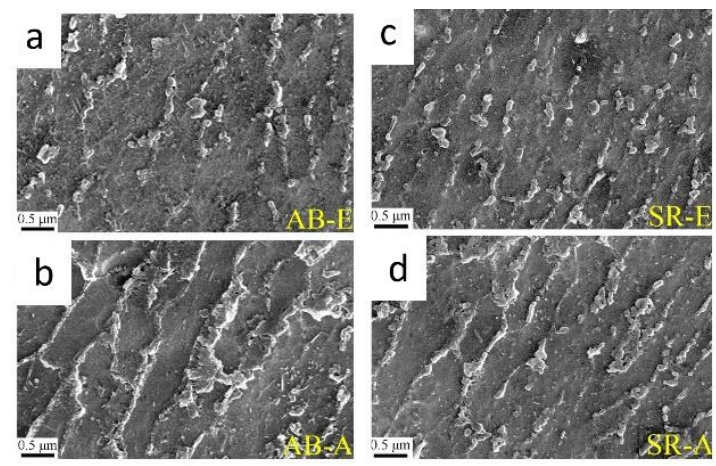

Fig. 3. SEM images. see Fig. 1 for the location in which the samples were fabricated.

that can explain the differences in densities as presented in Table 1. The increased porosity can affect the dynamic flow and crack propagation. It is plausible to assume that a pore or a defect can absorb the energy located at the tip of a running crack; therefore the pores act as an energy absorption system. For the AB samples, the difference in the ductility and peak 
flow stress can be explained by changes in the microstructure. In figure 3 high magnification SEM images are presented. The images present the bottom (Figs. 3a and 3c) and top (Figs. $3 \mathrm{~b}$ and $3 \mathrm{~d}$ ) of the $\mathrm{AB}$ and SR samples respectively. In the SEM images, the light gray color are the $\alpha-\mathrm{Al}$ and Si phases. The similarity in the $\alpha-\mathrm{Al}$ distribution in frames $\mathrm{a}, \mathrm{b}$ and $\mathrm{d}$ is clear. In frame $\mathrm{b}$ of figure 3 the $\alpha-\mathrm{Al}$ and Si phases are connected and there is very little gap between them. That picture is in correlation with the stress-strain curves presented in figure 2 . From this structure we assumed that the $\alpha$-Al and Si phases play an important role in the response of the material to dynamic load and crack propagation.

\section{Conclusions}

The present investigation focused on the effects of heated building platform and the influence of stress relief heat treatment on the dynamic mechanical properties of SLM AlSil0Mg. The samples were dynamically loaded by SHPB system followed by advanced microscopy analysis. We found that the heat from the building platform act as stress relief treatment to the samples. To validate this finding a comparison was done with samples that experienced controlled stress relief heat treatment. The SHPB and the SEM images strengthen that finding. The stress relief treatment reduced the variance in the microstructure, softened the material and changed the crack propagation. In the results obtained from the SHPB we note a decrease in the true stress as the true strain dynamically increases. We relate this observation to accumulative damage in the samples during the loading process. SEM analysis reveals different crack paths at different distances from the building platform. The changes in the $\alpha$-Al cells observed in the SEM analysis transform the crack propagation path from the melting pool boundaries to the somewhat stochastic paths along the sample.

\section{Acknowledgments}

B. Amir is supported by the Levtzion Scholarships of the council for higher education.

\section{References}

1. D. Buchbinder, H. Schleifenbaum, S. Heidrich, W. Meiners, J. Bültmann. Phys Procedia, 12, PART 1, (2011)

2. K. Kempen, L. Thijs, J. Van Humbeeck, J-P. Kruth. Phys Procedia. 39:439-446. (2012)

3. F. Calignano, D. Manfredi, E. P. Ambrosio, L. Iuliano, P. Fino, Int J Adv Manuf Technol.;67(9-12):2743-2751, (2013).

4. N. Read, W. Wang, K. Essa, M. M. Attallah, Mater Des. 65:417-424 (2015).

5. P. Ponnusamy, R. A. Rahman Rashid, S. H. Masood, D. Ruan, S. Palanisamy, Materials (Basel). 13 (19):4301. (2020)

6. B. Nurel, M. Nahmany, n. Frage, A. Stern, O. Sadot, Additive Manufacturing 22, 823833, (2018)

7. B. Amir, S. Samuha, O. Sadot. Materials (Basel) ;12(7):1143. (2019)

8. B. Amir, E. Grinberg, Y. Gale, O. Sadot, S. Samuha, Materials Science \& Engineering A (to be published) 